\title{
Signal processing of white-light interferometric low-finesse fiber-optic Fabry-Perot sensors
}

\author{
Cheng $\mathrm{Ma}^{*}$ and Anbo Wang \\ Center for Photonics Technology, Bradley Department of Electrical and Computer Engineering, Virginia Polytechnic \\ Institute and State University, Blacksburg, Virginia 24061-0111, USA \\ ${ }^{*}$ Corresponding author: cma1 @ vt.edu
}

Received 25 September 2012; revised 27 November 2012; accepted 27 November 2012; posted 28 November 2012 (Doc. ID 176854); published 4 January 2013

\begin{abstract}
Signal processing for low-finesse fiber-optic Fabry-Perot sensors based on white-light interferometry is investigated. The problem is demonstrated as analogous to the parameter estimation of a noisy, real, discrete harmonic of finite length. The Cramer-Rao bounds for the estimators are given, and three algorithms are evaluated and proven to approach the bounds. A long-standing problem with these types of sensors is the unpredictable jumps in the phase estimation. Emphasis is made on the property and mechanism of the "total phase" estimator in reducing the estimation error, and a varying phase term in the total phase is identified to be responsible for the unwanted demodulation jumps. The theories are verified by simulation and experiment. A solution to reducing the probability of jump is demonstrated. (C) 2013 Optical Society of America
\end{abstract}

OCIS codes: $\quad 060.2370,120.3180,120.2650,120.2230$.

\section{Introduction}

Low-finesse fiber-optic Fabry-Perot interferometric (FPI) sensors have been investigated for more than two decades. Both extrinsic and intrinsic FPIs (denoted as EFPIs and IFPIs, respectively) have sought extensive applications in displacement, temperature, strain, pressure, and acoustic sensing [1-드. In such applications, the change of the optical path difference (OPD), defined as twice the product of the cavity length and the refractive index of the cavity material, is monotonically or linearly related to the physical parameters being measured; as a result, the key to signal processing is to make an accurate measurement of the OPD. The reflection spectrum of the Fabry-Perot (FP) sensor is expressed as []

$$
I(\Phi)=2 R \frac{1-\cos (\Phi)}{1+R^{2}-2 R \cos (\Phi)},
$$

$1559-128 \mathrm{X} / 13 / 020127-12 \$ 15.00 / 0$

(C) 2013 Optical Society of America where $R$ is the reflectance of the cavity mirrors (assume identical reflectivity) and $\Phi$ denotes the total phase defined through $\Phi=k \cdot \mathrm{OPD}$, where $k$ is the wavenumber in vacuum. In the regime of "low finesse," the reflectances of the cavity mirrors are low and multireflections are neglected. Such low-finesse FP sensors are more cost-effective, and most importantly, they exhibit outstanding robustness in harsh environments [7]. For such sensors Eq. (1) is reduced to

$$
I(\Phi)=2 R[1-\cos (\Phi)]
$$

which appears sinusoidal with respect to $\Phi$, and $\Phi$ is linear with both OPD and $k$. In fringe counting mode, a fixed-wavelength laser (or several lasers at different but fixed wavelengths) is injected into the sensor and the reflected power is modified sinusoidally with the OPD (when $k$ is fixed) [8]. Unfortunately, the output is inherently periodic, so it provides only a relative OPD measurement. Absolute measurement is apparently more desirable and can be achieved using white-light interferometry (WLI), in 
which a broadband optical source is used. With WLI, the OPD is measured either by matching the cavity length of a reference interferometer [9] or by direct analysis of the reflected spectrum; the latter is the subject of this paper.

The schematic of the WLI sensing system is depicted in Fig. 1. The spectrum interrogation unit comprises a broadband source and a spectrometer (or a swept-laser spectrometer), which records the reflection spectrum of the FP sensor and sends the data to a processing unit (computer) for demodulation. The spectrum is sinusoidal with wavenumber $k$ according to Eq. (2). A variety of demodulation methods have been developed to calculate the OPD directly from the spectrum, and they can be classified into two categories.

The first type is based on direct frequency estimation and is denoted as TYPE I, which relies on the fact that the spectrum is a harmonic function of $k$, with OPD being the frequency. Estimating the frequency can be achieved by using a periodogram [i.e., by Fourier transform (FT)] or phase linear regression. A periodogram is mostly adopted in the estimation of the frequency by tracking the peak position in the FT of the spectrum [10-14]; this method is especially powerful for demodulation of multiplexed sensors. An alternative TYPE I approach relies on $\Phi=\mathrm{OPD} \cdot k$, where multiple $\Phi$ and $k$ values are obtained from the spectrogram, and consequently calculation of OPD is converted to frequency estimation by linear regression (LR). A simple but popular version uses only two points and usually employs peaks, valleys, or zero-crossing points $[1,3,15]$. This method offers low resolution and works better for situations where only a few peaks exist in the spectrum. If more peaks are available, it is straightforward to use more points to maximize information utilization. In such cases, multiple peak/valley points $[2,16]$ or all the spectral data $[17,18]$ are used for the LR.

The second type of algorithm, named TYPE II (total phase), also relies on the relationship $\Phi=\mathrm{OPD} \cdot k$. In a simple version, the spectral position $k$ of one peak is accurately determined, and the OPD is obtained by $\Phi / k$, where the value $\Phi$ of the peak is known if the corresponding fringe order is found. Without the knowledge of the fringe order there is an $2 \pi$ ambiguity in $\Phi$ [19]. In order to enable absolute measurement, an improved algorithm estimates the fringe order in the first step by FT or LR and subsequently determines the OPD by tracking one peak $[20,21]$. This strategy was extended further to utilize the whole spectrum [22]. Apart from estimating only the frequency component in the spectrograph as in TYPE I methods, this approach seeks to

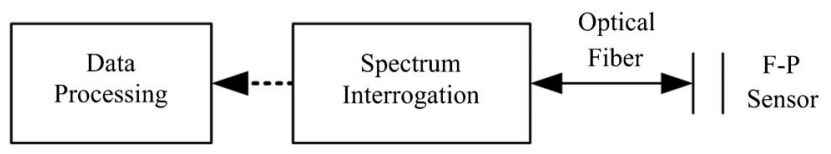

Fig. 1. Schematic of the fiber-optic WLI FP sensing system. estimate the total phase $\Phi$ at given spectral points $k$ (or vice versa). Similar tasks were also accomplished by iterative curve fitting [23-25].

Despite the success of these developed methods, problems still exist. It is widely accepted (and will be demonstrated later) that the TYPE II approach (total phase) is more accurate than the TYPE I (frequency estimation) in calculating the OPD. Unfortunately, because the total phase is periodic in nature, a $2 \pi$ ambiguity may occur during implementation, which leads to abrupt discontinuity (or "jumps") in the demodulated OPD [22-25] (it should be noted that jumps only exist in TYPE II estimations). To date, the cause of the jump is still not thoroughly understood, and consequently eliminating the jump is still not promising. Moreover, a theoretical framework is missing within which the performance of the different algorithms can be evaluated and compared. Due to these reasons, it is reasonable to conclude that the practicality and potentiality of the WLI algorithms are still not fully exploited.

This paper constructs a set of theories and criteria upon which the performance of the algorithms can be evaluated, and the cause of the jump problem is well explained (Section 2). We emphasize the role of an additional phase term that acts as a "hidden killer" to cause the jumps and propose a more appropriate definition of the total phase with the hope of maximizing noise reduction while maintaining minimum jump risk. In Section $\underline{3}$, the theories will be verified and discussed. Three WLI algorithms will be compared, and their pros and cons will be concluded. The physical cause of the additional phase will be further expounded and a solution to minimize the jump occurrence will be demonstrated.

\section{Theory}

\section{A. Background}

In deriving Eq. (1), a plane wave with normal incidence to the cavity mirrors is assumed. In a real application, the beam inside the cavity is diffraction limited and its divergence gives rise to a round-trip power coupling factor $v$, and accordingly Eq. (1) is modified to

$$
I(\Phi)=R \frac{1+\nu^{2}-2 \nu \cos (\Phi)}{1+R^{2} \nu^{2}-2 R \nu \cos (\Phi)}
$$

with $\nu<1 . I(\Phi)$ is periodic with $\Phi$ and can be subsequently expressed by a Fourier series as

$$
I(\Phi)=a_{0}+\sum_{n=1}^{\infty} a_{n} \cos (n \Phi)
$$

with Fourier coefficients $a_{n}$ (the sine terms are absent due to symmetry). It is shown that the intensity ratio between adjacent harmonics is (for $n>0$ )

$$
a_{n+1} / a_{n}=R \nu,
$$


which dictates that higher harmonics are orders of magnitude weaker than the fundamental component and can be neglected. As a result we are left with a pure sinusoidal signal, which after normalization appears to be

$$
I_{\mathrm{norm}}(\Phi)=1+\frac{2 \nu}{1+\nu^{2}} \cos (k \cdot \mathrm{OPD}+\pi) .
$$

The influence of the beam divergence is to reduce the signal strength by a factor of $2 \nu /\left(1+\nu^{2}\right)$, which is usually referred to as the fringe visibility.

The above discussion only takes into account the loss generated by the beam divergence. In fact, the divergence also distorts the wave front of the beam, which introduces another phase term $\varphi_{0}$ (additional phase) in the total phase $\Phi$ when the light is coupled back into the lead fiber [25]. Equation ( $\underline{6})$ is finally written in the following form (where $\varphi_{0}+\pi$ is combined into $\varphi_{0}$ ):

$$
I_{\text {norm }}(\Phi)=1+\frac{2 \nu}{1+\nu^{2}} \cos \left(k \cdot \mathrm{OPD}+\varphi_{0}\right),
$$

which best describes the spectrum of a real lowfinesse FP sensor. The TYPE I (frequency estimation) approaches calculate OPD directly from the density of the fringes, and thus the additional phase term $\varphi_{0}$ is ignored. On the contrary, this term needs to be considered in the TYPE II approach, due to its direct contribution to the value of the total phase, and is usually treated as a constant and precalibrated $[20,22]$. Nevertheless, this treatment is in most cases not suitable and will result in the demodulation jumps, as will be discussed later.

Most spectrometers provide spectral position readings in units of wavelength $(\lambda)$, which result in an uneven distribution of $k$ (i.e., $k=2 \pi / \lambda$ ). It is recommended that the spectrum be interpolated to yield an equal sampling interval in $k$ to take full advantage of standard signal processing techniques. The interpolation error is minimized if a "cubic spline" interpolation is used, and the associated interpolation error shows negligible influence on the final estimation quality, as will be demonstrated in Section 3.B.

We conclude that the OPD estimation problem is now converted to standard harmonic analysis. Before continuing the theoretical discussion, the scope of the subject matter needs to be further clarified. The signal is a discrete, real-valued sequence, with unknown parameters OPD (frequency) and $\varphi_{0}$ (phase) to be estimated. Because the data are real-valued, we define the normalized frequency by $\omega_{n}=\omega /\left(2 \omega_{s}\right)$, where $\omega_{s}$ is the angular sampling frequency, and thus

$$
\omega_{n}=\mathrm{OPD} \cdot \Delta k / \pi,
$$

where $\Delta k$ denotes the spectral sampling interval. As can be easily verified, for real systems Eq. (ㅁ) always yields a number $<0.1$, which suggests that the signal has "moderately low" frequency. The phrase "moderately low" is used to distinguish from "extremely low," in which the FT peak in the periodogram is nearly indistinguishable from the direct current (dc) component that standard frequency estimation methods (such as the ones to be introduced in Section 3) tend to generate large errors. In such cases, the method in [20] is suggested. If the FT peaks are clearly resolvable in the Fourier domain, a classical nonparametric periodogram yields the best performance (i.e., the maximum likelihood estimator) [26]; this condition is satisfied for most cases and is the subject of this paper. More specifically, this paper is confined to single harmonic analysis. For multitone analysis a filter is usually used to select the corresponding frequency component; however, the problem is complicated by issues such as crosstalk among different sensors, and the lower bounds of the estimators need to be modified [26]. If multiple harmonic components closely coexist with deteriorated resolution on the periodogram, parametric methods can be applied [27]. In some applications, the signal is amplitude-modulated by an envelope stemming from the source spectrum. The envelope can be removed by precalibrating the source spectrum or by an interpolation process [21,22]. In sum, after the removal of the envelope and the DC component, the signal is expressed as

$$
I_{n}=A \cos \left(\mathrm{OPD} \cdot k_{n}+\varphi_{0}\right)+W_{n},
$$

where $A$ is the amplitude, $k_{n}$ is the fixed sampling wavenumber, and $I_{n}$ is the measured intensity at $k_{n}$, which is contaminated by zero-mean white Gaussian noise $W_{n}$ with power $\sigma^{2}=\operatorname{var}\left[W_{n}\right](\operatorname{var}[\bullet]$ denotes the variance). The quantities in Eq. (9) are all real-valued and $n=1,2, \ldots N$ ( $N$ is the length of the data). OPD and $\varphi_{0}$ will be the parameters to be estimated; their corresponding estimation values are denoted as OPDD and $\hat{\varphi}_{0}$, respectively. The signalto-noise ratio (SNR) is defined as $S=A^{2} /\left(2 \sigma^{2}\right)$ [28]. The beginning wavenumber is defined as $k_{0}$ and subsequently $k_{n}=k_{0}+(n-1) \Delta k$, and we define a central wavenumber

$$
k_{c}=\left(k_{1}+k_{N}\right) / 2 \approx k 0+N \Delta k / 2,
$$

which is heavily used throughout the rest of the paper.

Two WLI systems will be applied for simulation and experimental demonstration of the theories. Their system parameters are listed in Table $\underline{1}$.

\section{B. Theories}

\section{Cause of the Jump (I): the Constant $\varphi_{0}$} Assumption

For the TYPE II (total phase) method, if the demodulation algorithm assumes that $\varphi_{0}$ is a constant, the jump problem becomes inherent when in fact $\varphi_{0}$ changes during the measurement and the change exceeds $\pi$. A detailed analysis of this phenomenon is found in [29]. This can be intuitively understood 
Table 1. List of Key Parameters of the WLI Systems Used in the Research

\begin{tabular}{lccccccc}
\hline $\begin{array}{l}\text { System } \\
\text { Number }\end{array}$ & Source & Spectrometer & Wavelength & & & \\
I & LED & Range $(\mathrm{nm})$ & $N$ & $k_{0}\left(\mu \mathrm{m}^{-1}\right)$ & $\Delta k\left(\mathrm{~m}^{-1}\right)$ & Remarks \\
II & OceanOptics, Inc., USB2000 & $715.88-980.64$ & 2048 & 6.4072 & 1157.6 & Multimode fiber based \\
& Micron Optics, Inc., swept laser spectrometer with & $1520-1570$ & 20,000 & 4.0020 & 6.5826 & Single-mode fiber based \\
& built-in source, Si-720 & & & & & \\
\hline
\end{tabular}

as follows. While $\varphi_{0}$ shifts, as long as the drift is less than $\pi$, the algorithm can find a nearest fringe order $\left(N_{0}\right)$ by a rounding process [20]. However, when the shift is beyond $\pi$, the nearest fringe order suddenly moves to $N_{0} \pm 1$, and the jump occurs. As a result, assumption of a constant $\varphi_{0}$ should be avoided to prevent jumps.

\section{Total Phase Estimation with Varying $\varphi_{0}$}

As stated in the previous subsection, when applying the TYPE II approach (to calculate the total phase), $\varphi_{0}$ cannot be treated as a constant. Instead, it should be estimated along with the OPD, and the total phase can be estimated by [29]

$$
\hat{\Phi}=k_{c} \cdot \mathrm{OPD}+\hat{\varphi}_{0},
$$

where OPDD and $\hat{\varphi}_{0}$ are estimated by the TYPE I algorithm and subsequently an estimation of OPD with better resolution is given by

$$
\mathrm{OPD}_{\text {tot }}=\hat{\Phi} / k_{c} \text {. }
$$

The subscript tot denotes TYPE II OPD estimation by total phase. A major advantage of TYPE II estimation is its superior noise reduction capability. In Section 2.B.3 and Appendix A, the noise reduction mechanism of the TYPE II approach will be analyzed. Moreover, using total phase also benefits from a reduction of bias and demodulation nonlinearity, as will be discussed in Sections 3.C.1 and 3.E.1.

Using Eq. (11), the prerequisite of constant $\varphi_{0}$ is removed. Typically, $\varphi_{0}$ depends on OPD in a deterministic way and can be calibrated within the measurement range [e.g., by polynomial fitting $\varphi_{0}=\mathbf{P}(\mathrm{OPD})$ ]. In real practice, one needs to estimate OPD and $\hat{\varphi}_{0}$ in the first step. Similar to $\Phi, \varphi_{0}$ is a phase term and is consequently subject to $2 \pi$ ambiguity. If $\varphi_{0}$ is precalibrated [meaning the relationship of $\varphi_{0}=\mathbf{P}(\mathrm{OPD})$ is found], one can calculate the expectation value of $\varphi_{0}$ at the given OPD (calculated in advance via TYPE I estimation $)$ as $\tilde{\varphi}_{0}=\mathbf{P}(\mathrm{OPD})$ and set a $2 \pi$ range $\left[\tilde{\varphi}_{0}-\pi, \tilde{\varphi}_{0}+\pi\right]$ to determine $\hat{\varphi}_{0}$. As will be shown in Section 3.E.2, this technique can reduce the probability of jump by several orders of magnitude.

\section{Lower Bounds}

For an estimator, performance can be evaluated by the mean square error (MSE):

$$
\mathbf{E}\left[(\hat{\theta}-\theta)^{2}\right]=\operatorname{var}[\hat{\theta}]+\operatorname{bias}^{2}[\hat{\theta}],
$$

where $\mathbf{E}$ denotes the average and $\hat{\theta}$ is the estimated value of the parameter $\theta$. In general the estimator is assumed to be unbiased, and the MSE reduces to the variance of the estimator. However, unbiasedness is not a straightforward assumption and will be discussed in detail in Section 3.C.1. In any circumstances, the variance of the estimator cannot be arbitrarily small, the lower bound of which is defined by the Cramer-Rao bound (CRB). Here, without proof, the CRBs for the frequency and phase estimations in WLI are given; the derivations can be found in [30]. Defining $P=N(N-1) / 2, Q=N(N-1)(2 N-$ 1) $/ \overline{6,} n_{0}=k_{0} / \Delta k$, and $Y=n_{0}^{2} N+2 n_{0} P+Q$,

- The CRB of OPD estimation with unknown $\varphi_{0}$ is (OPD of TYPE I)

$$
\operatorname{var}[\mathrm{OPD}]=\frac{12}{S \cdot(\Delta k)^{2} N\left(N^{2}-1\right)}
$$

- The CRB of OPD estimation with known $\varphi_{0}$ is (this is an approximation of the CRB of TYPE II OPD; see Appendix $\underline{\text { A) }}$

$$
\operatorname{var}[\mathrm{OPD}]=\frac{1}{S \cdot(\Delta k)^{2} Y} .
$$

- The CRB of $\varphi_{0}$ estimation with unknown OPD is $\left(\varphi_{0}\right.$ of TYPE I)

$$
\operatorname{var}\left[\hat{\varphi}_{0}\right]=\frac{12 Y}{S \cdot N^{2}\left(N^{2}-1\right)}
$$

- The CRB of $\varphi_{0}$ estimation with known OPD is ( $\Phi$ of TYPE II)

$$
\operatorname{var}\left[\hat{\varphi}_{0}\right]=\frac{1}{S \cdot N}
$$

For WLI systems, both OPD and $\varphi_{0}$ are unknown. Equation (14) defines the lower bound of the OPD estimation variance for the TYPE I algorithms ("frequency estimation"); the lower bound of phase estimation variance of TYPE I methods is given by Eq. (16). Equation (17) is the CRB of $\Phi$ estimation [total phase of TYPE II, Eq. (11)], and accordingly the TYPE II OPD estimation $\left(\hat{O P} D_{\text {tot }}\right)$ has a variance limit of 


$$
\operatorname{var}\left[\hat{\mathrm{OP}} \mathrm{D}_{\mathrm{tot}}\right]=\frac{1}{S \cdot N \cdot k_{c}^{2}}
$$

To demonstrate that Eq. (17) is the CRB of $\hat{\Phi}$, we take the variance on both sides of Eq. (11) and notice that when the frequency OPD is known, the variances of $\hat{\Phi}$ and $\hat{\varphi}_{0}$ coincide, which sets the lower bound of the total phase estimation. A more detailed explanation of the noise reduction mechanism of the total phase approach is given in Appendix A. The benefit of using the total phase is clear when Eqs. (18) and (14) are compared. The root mean square (rms) error of the direct frequency estimation is much larger than the total-phase-based OPD estimation by a factor of [by taking the square root of Eq. (14)/ Eq. (18)]

$$
G=2 \sqrt{3}\left(\frac{n_{0}}{N}+\frac{1}{2}\right) .
$$

For WLI System I, it is calculated that $G \approx 11.09$, and for WLI System II, $G \approx 107.04$. This predicts that the OPD estimation resolution using total phase is approximately 10 and $20 \mathrm{~dB}$ better than the direct frequency estimation for Systems I and II, respectively. More benefits of using total phase will be further discussed in Section 3.E.1. In general, if high resolution is required, total phase demodulation is recommended. However, one major drawback of the approach is the potential of the demodulation jumps (there is no jump problem associated with the TYPE I method).

It should be noted that the fringe visibility (and hence the SNR) is OPD dependent [25]. Accordingly, for applications where the OPD is subject to large change, the CRBs should be treated as a function of the OPD.

4. Cause of the Jump (II): Estimation Noise of $\varphi_{0}$ The probability density function (PDF) of $\hat{\varphi}_{0}$ is a Gaussian with mean $\varphi_{0}$ and variance $\sigma_{p}^{2}$ [the lower bound of the variance is defined by Eq. (16)] and is expressed as

$$
f\left(\hat{\varphi}_{0} ; \varphi_{0}, \sigma_{p}^{2}\right)=\frac{1}{\sigma_{p} \sqrt{2 \pi}} \exp \left[-\frac{\left(\hat{\varphi}_{0}-\varphi_{0}\right)^{2}}{2 \sigma_{p}^{2}}\right] .
$$

As discussed in Section 2.B.2, a $2 \pi$ range is assigned to $\varphi_{0}$ in order to unambiguously determine its value. A jump event will occur if $\hat{\varphi}_{0}$ falls out of the assigned range; this would lead to addition or subtraction of $2 \pi$ multiple times in order to force $\hat{\varphi}_{0}$ back to the given range. The probability of jump is minimized if the range is centered at $\varphi_{0}$, where the probability is defined by the following inequity:

$$
P_{\text {jump }} \geq 2 F(f ;-\pi)=2 \int_{-\infty}^{-\pi} f\left(\hat{\varphi}_{0} ; 0, \sigma_{p}^{2}\right) d \hat{\varphi}_{0},
$$

where $F$ denotes the cumulative distribution function. It should be noted that in principle the jump cannot be eliminated [the value of Eq. (21) is always $>0$ ]; however, it can approach zero if $\sigma_{p}$ is small and the $2 \pi$ range is appropriately selected. The jump probability minimizes when the estimator reaches the CRB and the $2 \pi$ range is centered at $\varphi_{0}$. One can thus estimate the jump probability by Eqs. (16) and (21). The probability escalates rapidly if $\sigma_{p}$ is large and/or the range is not centered at $\varphi_{0}$; we will illustrate this situation in more detail in Section 3.E.2.

\section{Discussion}

\section{A. WLI Algorithms}

1. Periodogram (Fast Fourier Transform Method)

The maximum likelihood estimator of a single harmonic is the periodogram, which can be regarded as the best algorithm $[26,30]$. However, this method has been considered to yield poor accuracy when it is applied in WLI. The reasons for this belief are the following: (1) a periodogram was used as a TYPE I estimator in the past, i.e., only OPDD was estimated. As a result of the discussion in Section 2.B.3, compared with the TYPE II approaches, the resolution of a periodogram is worse. (2) The implementation of the algorithm is not optimized.

Because the signal is real, its FT is symmetric about zero and only $N / 2$ data points are effective in the periodogram. This basically dictates that the normalized frequency resolution is limited to $2 / N$. To improve the resolution, zero padding is widely adopted to increase $N$ [13]. Taking the derivative of Eq. (8) on both sides and denoting the frequency resolution as $d \omega_{n}=2 / N$, it is found that in order to resolve a frequency difference of $\delta \mathrm{OPD}$, the number of points needed is

$$
N=\frac{\lambda_{c}^{2}}{\delta \mathrm{OPD} \cdot \Delta \lambda},
$$

in which $\lambda_{c}$ is the central wavelength and $\Delta \lambda$ is the wavelength interval of the spectrometer. To achieve a resolution of $\delta \mathrm{OPD}=1 \AA$, for WLI System I, $N=$ $7.4 \times 10^{7}$, and for WLI System II, $N=9.6 \times 10^{9}$. The required data size will lead to an unrealistic computational load and result in extremely low efficiency. To improve its efficiency, it is recommended that following an approximate estimation of the FT peak position with relatively short $N$, the normalized frequency in Eq. (8) is further determined by the Newton-Raphson method to maximize [26, $\underline{30}]$

$$
\left|A\left(\omega_{n}\right)\right|=\left|\sum_{n=0}^{N-1} I_{n} \exp \left(-i \cdot \pi n \omega_{n}\right)\right|,
$$

and the estimations of OPD and $\varphi_{0}$ are obtained by

$$
\mathrm{OPD}=\hat{\omega}_{n} \pi / \Delta k
$$




$$
\hat{\varphi}_{0}=\arg \left[\exp \left(-i \mathrm{OPD} \cdot k_{0}\right) A\left(\hat{\omega}_{n}\right)\right] .
$$

This method is expected to yield the maximumlikelihood estimation to reach the CRB. It is abbreviated as the fast Fourier transform (FFT) method and will be discussed further in the following subsections.

\section{Linear Regression Method and Peak Tracking Method}

In the LR method, $\Phi$ of all the data points are calculated by solving the angle of the analytical signal, followed by a linear fitting to their corresponding spectral positions $k_{n}$. Details of the method can be found in [22]. Equation (13) in [22] is used to estimate the OPD and $\varphi_{0}$.

Instead of using all the spectral points for LR, the peak tracking (PT) method uses only the peak positions in the spectrum. Corresponding details can be found in [29], and Eqs. (21) and (22) in [29] are used to determine the OPD and $\varphi_{0}$. At first glance the accuracy of PT should be lower than LR, because only a small portion of the spectral data (only the peak points) are used; however, this is not true, because actually all the points contribute to the determination of the peak positions.

For the LR and PT methods, it is important to use a bandpass filter to clean the signal in advance to increase the breakdown threshold, which is defined as an SNR value beyond which the error of the estimator abruptly becomes significantly larger than the CRB [22]. It is found that an effective order of the filter is $N_{f}=N / 10$. If $N_{f}$ is too small, the noise reduction is not sufficiently effective; on the other hand, if $N_{f}$ is too large, the effective length of the data is sacrificed to an extent that the CRB is apparently lifted. It is also important to shift the filtered data back to $N_{f} / 2$ points to compensate the filtering delay, or a large phase bias would occur.

\section{B. Evaluation of the Algorithms}

The algorithms in Section 3.A were evaluated by comparison with the corresponding CRBs using computer simulation. In the simulation, an FP cavity with $\mathrm{OPD}=200 \mu \mathrm{m}$ was assumed, and the parameters of WLI System I were used. White Gaussian noise was added to the sensor spectrum according to the required SNR. At each SNR level, 1000 sets of spectral data were generated, and the variances of the FFT, LR, and PT methods were calculated for both TYPE I and TYPE II [by using Eqs. (11) and (12)] estimations. Plotted together are the corresponding CRBs. In Fig. 2(a), the CRB for frequency estimation with known phase is plotted, which is sufficiently accurate as the CRB for a TYPE II OPD estimator (see Appendix A). Because the estimators were assumed to be unbiased, the simulation used their standard deviation as the rms error. The discussion on estimator bias is given in Section 3.C.1. As demonstrated by Fig. 2, all three algorithms approach the CRBs nicely. It should be noted that the

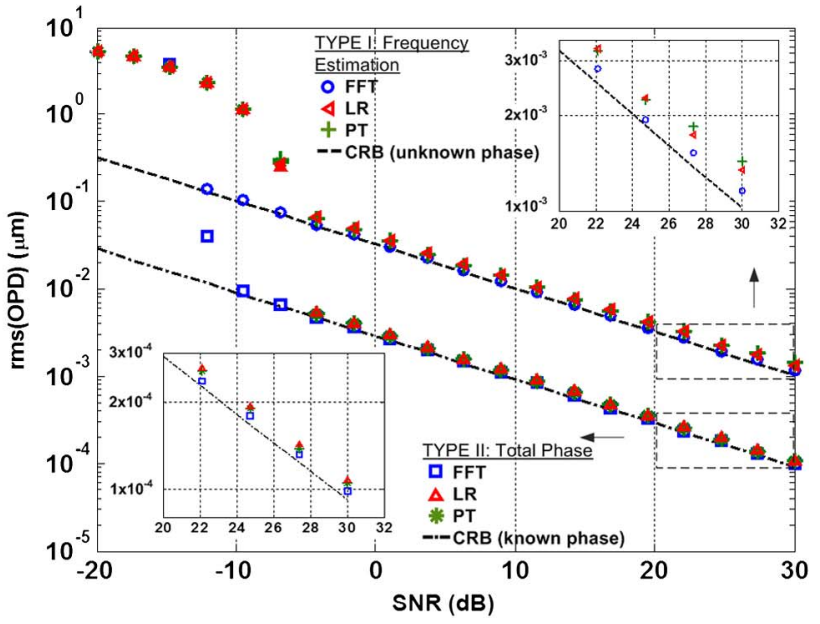

(a)

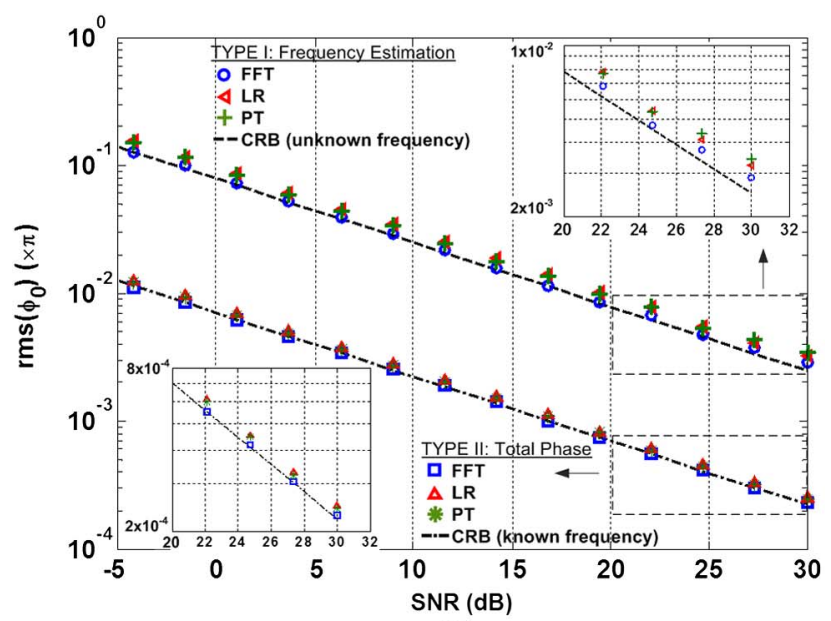

(b)

Fig. 2. (Color online) Performance evaluation of the FFT, LR, and PT methods: (a) OPD estimation and (b) $\varphi_{0}$ estimation. Plotted together in both figures are the standard deviations of both the TYPE I and TYPE II estimators. The CRBs for the corresponding variances are coplotted. Insets are zoomed-in views of the lines, which provide better visibility of the algorithms' performance.

input spectrum was evenly sampled in wavelength. Reaching the CRB demonstrated the effectiveness of the interpolation process that evenly sampled the spectrum in wavenumber. The FFT method, as expected, shows the best performance, whereas the LR and PT methods demonstrate nearly identical performances. The threshold of the LR and PT methods occurs at SNR $\sim-5 \mathrm{~dB}$, and the FFT approach shows a lower threshold, below $-10 \mathrm{~dB}$, which suggests better immunity to noise. Reaching the CRB is a manifestation of the performance optimization. In other words, once an algorithm approaches the CRB, it reaches the ultimate limit given the amount of information. Any other WLI algorithms can be evaluated against the CRB under the same framework.

\section{Comparison of the Algorithms}

\section{Estimator Bias}

In the previous discussions, the estimators are assumed to be unbiased [30], which is in fact not true 
for the current situation. The reason is closely related to the properties of the WLI signal. As noted in Section 2.A, the harmonic under analysis is real-valued and bears a very low frequency. Unlike a complex-valued signal (analytic signal), the real signal is symmetric about zero on the Fourier domain. When the harmonic frequency is low, the positive and negative peaks associated with the harmonic are very close to each other. As the sequence is of finite length, spectral leakage will take place [28,31], manifesting as sidelobes near the FT peaks. The sidelobes of the negative peak interfere with the positive peak and slightly change its spectral position. The modification is small, but a noticeable bias is incurred when high accuracy is required.

Windows can be applied to the data to effectively suppress the intensity of the sidelobes. The performances of different windows are compared by computer simulation, in which the cavity length of the FP sensor was continuously swept from 3 to $100 \mu \mathrm{m}$, with the cavity refractive index set to unity. During the simulation no noise was added, and the WLI System I parameters were used in the study. The TYPE I OPDs estimated using LR, FFT, and windowed FFT (with a Blackman-Harris window) were obtained, and the bias of each estimator at the given cavity length was found by comparing with the true value. The results are shown in Fig. 3. A common feature shown is the tendency toward a short cavity, where the influence of spectral leakage surges rapidly for all the estimators, resulting in an increased bias. Another feature is the bias's pattern that oscillates with the cavity length, which is apparently a signature of the sidelobes. For TYPE I estimation, FFT and LR show unsatisfactorily large biases. Even at a cavity length of $100 \mu \mathrm{m}$, both show a maximum bias of $\sim 10 \mathrm{~nm}$. The oscillatory pattern will promote demodulation nonlinearity. The effectiveness of

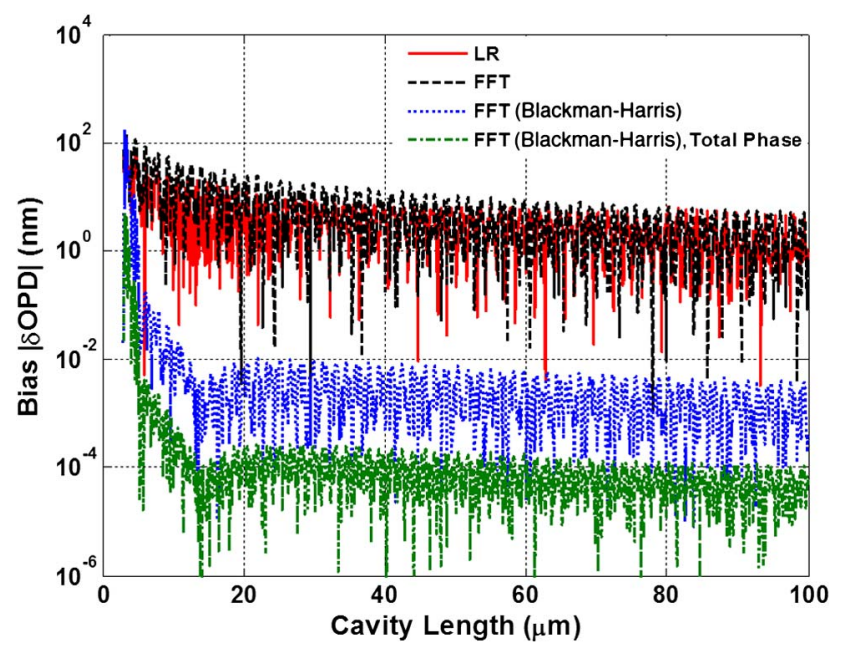

Fig. 3. (Color online) Absolute value of OPD bias versus cavity length plotted for TYPE I LR, FFT, and windowed FFT (Blackman-Harris) estimators. The result for the TYPE II estimator using FFT (Blackman-Harris) is coplotted, which demonstrates superior bias suppression. windowing to suppress the bias is demonstrated. As shown by the dotted curve, application of Blackman-Harris window leads to a bias suppression ratio over $30 \mathrm{~dB}$; the maximum bias was reduced below $10 \mathrm{pm}$ for cavity lengths from 10 to $100 \mu \mathrm{m}$.

During the simulation, $\varphi_{0}$ in the spectrum was set to zero; as a result the TYPE II OPD estimator [Eqs. (11) and (12)] became unbiased (otherwise the TYPE II estimator has an constant bias, which will be discussed in Section 3.E.1). It is found that the TYPE II estimator showed superior bias reduction, which suppressed the bias further by another $\sim 15 \mathrm{~dB}$. The maximum bias for cavity length ranging from 10 to $100 \mu \mathrm{m}$ was uniformly below $1 \mathrm{pm}$, which is of significant importance for high-accuracy applications.

\section{Comparison of the Algorithms}

In Section 3.B the performances of the algorithms are evaluated by comparing with the CRBs. In the previous demonstration only the variance was considered. According to Eq. (13), the MSE also includes a bias term; as discussed in the preceding section, the estimators are generally biased, which calls for reexamination of their performance. Comparison was made among FFT, LR, and windowed FFT (with a Blackman-Harris window); for both TYPE I and TYPE II estimations by computer simulation, the simulation assumes WLI System I parameters. In order to make the influence of the bias more visible, the OPD of the simulated spectrum was shortened to $60 \mu \mathrm{m}$. At each SNR level, the rms error was calculated based on comparison of the estimated and true OPD values with 1000 observations. The results are plotted in Fig. 4. The rms errors of the TYPE I LR and FFT estimators diverge significantly from the CRB for SNR $>10 \mathrm{~dB}$, which is attributed to a big bias. The curve corresponding to windowed FFT in the TYPE I group follows the CRB nicely in the whole SNR range except for a uniform $\sim 3 \mathrm{~dB}$ degradation. This is attributed to the larger equivalent noise bandwidth associated with the window used [31]. For the TYPE II estimators, FFT without windowing shows the worst performance (diverging significantly from the CRB starting at SNR $\sim 10 \mathrm{~dB}$ ). For the TYPE II LR estimator, significant divergence from the CRB happens above SNR $\sim 25 \mathrm{~dB}$. The performance of the windowed FFT is consistent in the complete SNR range. According to the simulation, for the TYPE I estimation, windowed FFT is preferred if SNR exceeds $20 \mathrm{~dB}$. For the TYPE II estimation, for SNR > $30 \mathrm{~dB}$, the windowed FFT has the best performance; otherwise the LR method is sufficient. For applications having a high SNR and requiring a high accuracy, the TYPE II windowed FFT is the best choice. The simulation predicts that for WLI System $\mathrm{I}$, at $\mathrm{SNR}=40 \mathrm{~dB}$, the rms estimation error is $\sim 50 \mathrm{pm}$ if TYPE II FFT with a Blackman-Harris window is used.

The computation complexity of the FFT, PT, and LR estimators were compared in terms of 


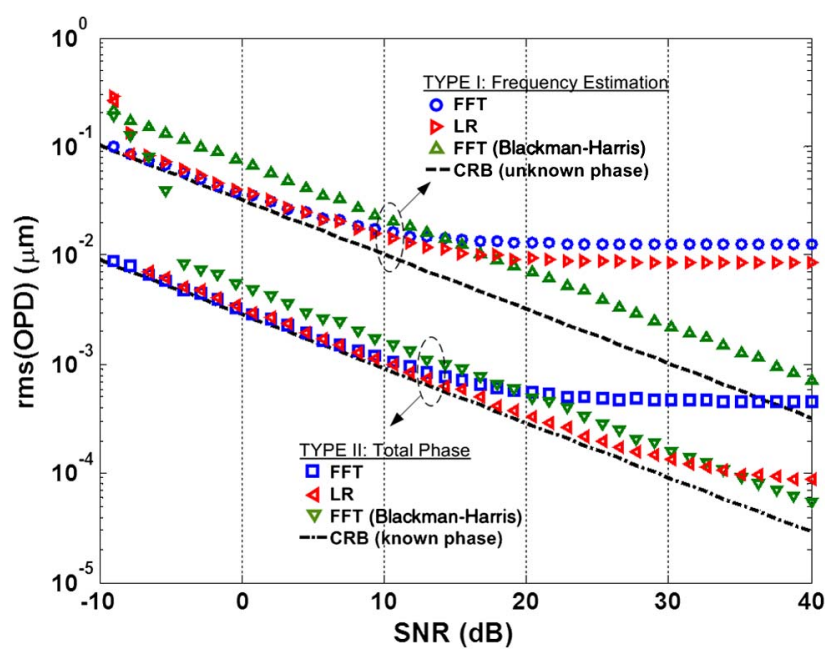

Fig. 4. (Color online) Performance comparison of the LR, FFT, and windowed FFT (Blackman-Harris). The rms error includes the contributions from both the estimator variance and bias. The windowed FFT for both TYPE I and TYPE II estimations manifests superior performance in bias reduction, at the expense of a $\sim 3 \mathrm{~dB}$ increase in the rms error.

computation time. The time was measured by averaging over 1000 executions and as a function of data length $(N)$, and the result is plotted in Fig. $\underline{5}$. The investigated algorithms show that the computation time linearly increases with $N$. The LR and PT methods have nearly identical complexity, while the complexity for the FFT method is significantly higher. The calculated complexity can be influenced by a number of factors, such as the CPU speed and programming skills. However the significantly higher complexity of the FFT estimator is as expected [32].

\section{OPD-Dependent Additional Phase}

We discussed in Section 2.B.1 that the "constant $\varphi_{0}$ " assumption will intrinsically lead to demodulation

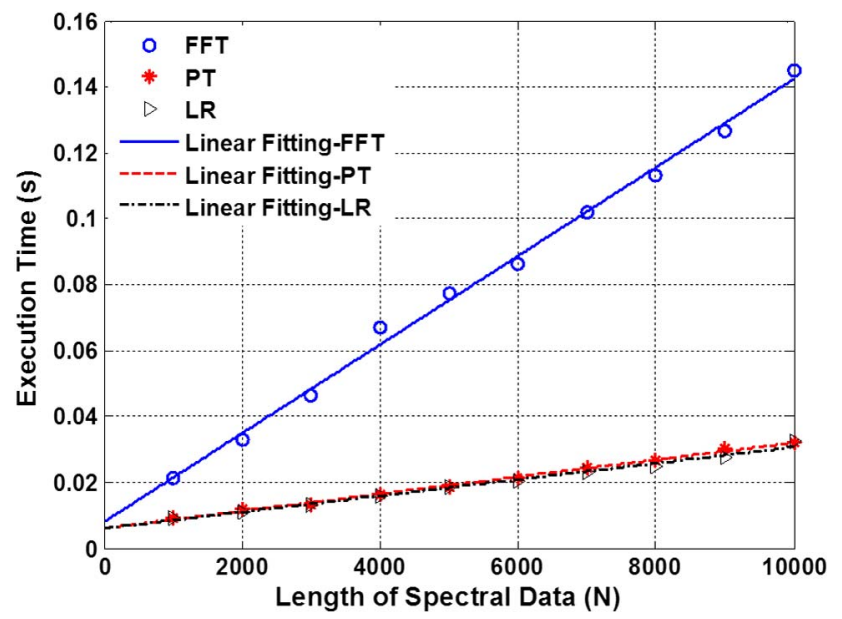

Fig. 5. (Color online) Measured computation complexity in terms of execution time, plotted as a function of data length $(N)$. The FFT, PT, and LR methods are compared to demonstrate a linear relationship with $N$. The complexity of FFT is the highest, while the complexities of PT and LR are almost identical. jumps if TYPE II estimation is made. We concluded from numerous experimental observations that the $\varphi_{0}$ term for both EFPI and IFPI is, in general, usually a function of OPD. Being a function of OPD makes the $\varphi_{0}$ calibratable, which is the key to reducing the probability of jumps, as will be discussed in Section 3.E.2. In this subsection some major causes for the OPD-dependence of $\varphi_{0}$ will be introduced; nevertheless, factors not included in this subsection may also cause a changing $\varphi_{0}$.

\section{Wave Front Distortion}

The $\varphi_{0}$ change due to wave front distortion is an inherent property of the FP cavity. In other words, unlike other factors, it is a "built-in" signature of the cavity and is unremovable by any hardware or software. At the cavity interface, the optical beam will be diffracted from a fiber mode into either a free-space mode (for an EFPI) or other fiber modes (for an IFPI). During propagation, the wave front of the cavity mode will become distorted, and a nonzero phase term will be generated when the wavefront-distorted beam is recoupled back into the lead-in fiber. Intuitively, the greater the distortion is, the larger the phase term; as the distortion is OPD-dependent, so is the induced phase. For EFPIs, detailed analysis can be found in $[25,33,34]$, and the case for IFPIs was studied in [35]. In general, for an EFPI, the phase term caused by wave front distortion is small and changes moderately with OPD; for an IFPI, due to multimode excitation, this term could be large and change more rapidly. It should be noted that multiple modes may still exist even when a single-mode fiber is used for the relatively short FP cavity as long as the single-mode fiber is different from the input single-mode fiber.

\section{Material Dispersion}

If the FP cavity is filled by a material with nonzero chromatic dispersion, and when the spectral bandwidth of the light detected is large (e.g., WLI System I), the material dispersion can cause a "chirp" in the spectrum that finally amounts to a spurious phase term. The material dispersion is described by the Sellmeier model; the coefficients of the model are all temperature-dependent. For a temperature sensor, changing the temperature subsequently changes the OPD, while the Sellmeier coefficients simultaneously vary to cause a changing $\varphi_{0}$. This was simulated using the parameters of WLI System I and a silica wafer with an initial thickness of $70 \mu \mathrm{m}$. Dispersion of the wafer was modeled by the temperaturedependent Sellmeier model with parameters from [36]. During the simulation, temperature was increased from $20^{\circ} \mathrm{C}$ to $1300^{\circ} \mathrm{C}$, and the linear coefficient of thermal expansion was set to $8.5 \times 10^{-6}$. The $\varphi_{0}$ term was calculated using a BlackmanHarris-windowed FFT and is plotted as the solid curve in Fig. $\underline{6}$. The agreement of the simulation result with the experiment will be given later in Section 3.E.2 (Fig. 8). 


\section{Fixed-Pattern Noise}

A pixel-sensor (such as a CCD) usually bears fixedpattern noise (FPN) due to uneven pixel-to-pixel sensitivity [37]. Some spectrometers (such as the spectrometer used in WLI System I) are built with such devices. Typically the FPN is small enough for accurate spectral analysis; however, for sensitive OPD measurement, the influence from the FPN can be noticeable. The OPD-dependent phase term $\varphi_{0}$ can be understood as follows. The spectrum of the FP sensor with the FPN is written as (with timedependent noise excluded)

$$
I_{n}=A \cos \left(\mathrm{OPD} \cdot k_{n}+\varphi_{0}\right)+\bar{W}_{n},
$$

where $\bar{W}_{n}$ represents the FPN. The Fourier domain spectrum is thus expressed as

$$
\tilde{I}_{k}=\mathbf{F F T}_{k}\left(I_{n}\right)=S_{k}+\tilde{w}_{k},
$$

where $S_{k}$ is the FT of the harmonic signal and $\tilde{w}_{k}$ is the FT of the FPN. A major difference between the FPN and normal noise is that $\tilde{w}_{k}$ is fixed for each Fourier bin. When the OPD changes, the FT peak position scans continuously, and the interference from $\tilde{w}$ is dependent on the peak position, so the OPD estimation error becomes OPD-dependent. This OPD error is finally translated to a phase error via Eq. (25). The FPN-induced phase shift always shows up with an oscillating pattern (with respect to OPD) with a period roughly equal to $\lambda_{c}$ (the central wavelength). This can be easily understood by recalling that after the OPD shifts $\lambda_{c}$, the spectrum nearly recovers, and the influence by the FPN will consequently repeat. The FPN-induced phase was simulated by adding a fixed white Gaussian noise to

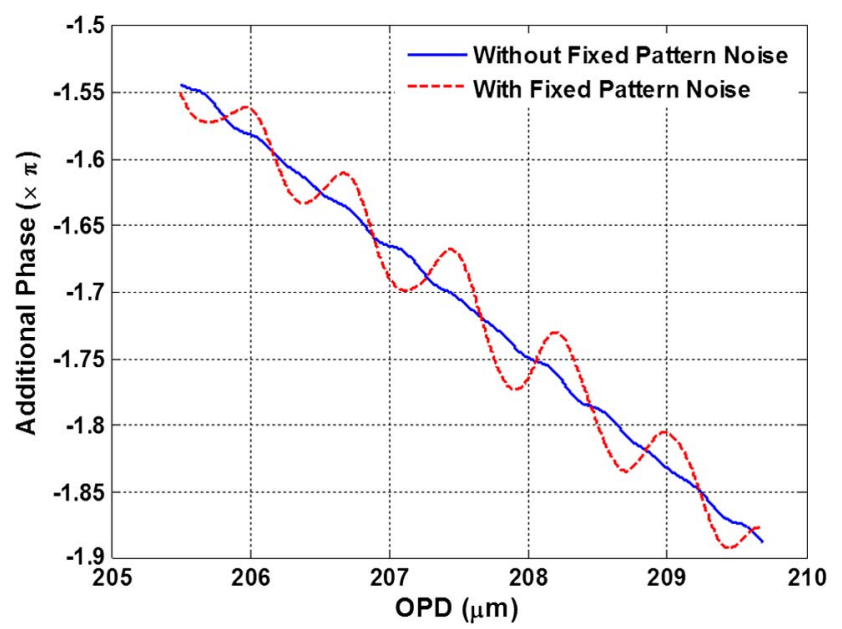

Fig. 6. (Color online) Computer-simulated phase term $\varphi_{0}$ caused by material dispersion (solid curve) and FPN (dashed curve). The dispersion of a $70 \mu \mathrm{m}$ thick silica wafer was modeled by the temperature-dependent Sellmeier model. WLI System I, together with Blackman-Harris windowed FFT, was used for signal demodulation. For the simulation of FPN, the applied white Gaussian noise yields $\mathrm{SNR}=12 \mathrm{~dB}$. the spectrum acquired by WLI System I, which resulted in an equivalent $\mathrm{SNR}=12 \mathrm{~dB}$. This noise was added upon the spectra that were previously used to simulate and plot the dispersion-related phase (Fig. 6, solid curve), and the final results are shown on the Fig. 6 as the dashed curve. The simulated influence indeed shows up as an OPDdependent oscillatory phase term.

\section{Finite Sampling Rate}

If the spectrometer has a finite sampling rate (FSR) across the full spectral range (e.g., WLI System II; the laser scanning rate is $5 \mathrm{~Hz}$ ), the FSR will couple with the OPD changing rate to generate an additional phase. The added phase can be expressed as $[\underline{29]}$

$$
\Delta \varphi_{0} \approx\left(\frac{d O P D}{d t}\right) \cdot \frac{k_{c}^{2}}{f_{s} N \Delta k}
$$

where $f_{s}$ denotes the scanning frequency and the derivative term represents the OPD changing rate. Among all the factors that induce the OPDdependent $\varphi_{0}$, this FSR-induced phase is the most difficult to remove, because it depends on the instantaneous OPD changing rate, and thus cannot be precalibrated.

\section{E. More on Total Phase}

\section{Characteristics of the Estimated Total Phase}

The benefit of TYPE II estimation is multifold. First, as was demonstrated previously, the TYPE II estimators have superior noise reduction capability. The rms error reduction factor for OPD is given in Eq. (19), and the CRB of the TYPE II OPD estimator approaches the CRB of OPD estimation with known phase. Second, as was shown in Section 3.C.1, the estimation bias of the TYPE II estimator is much lower than that of the TYPE I. More generally, the total phase estimation, $\hat{\Phi}$, follows the real OPD change more linearly than OPD. At first glance, the definition of TYPE II OPD estimation [Eq. (12)] introduces a bias term [Eq. (11), $\hat{\varphi}_{0}$ term). In fact, it is the inclusion of this term that reduces the demodulation nonlinearity. In real applications, the projection from the real OPD change to the demodulated OPDD (TYPE I) change is always not perfectly linear. This is caused by the nonlinearly changing $\varphi_{0}$ as discussed in Section 3.D. Interestingly, the nonlinearity that $\hat{\varphi}_{0}$ experiences is always accompanied by a proportional OPD change in the opposite direction (that is why OPD is not quite linear); however, the outcome is a cancellation of the nonlinearity in the total phase, which adds the two oppositely diverging terms. In sum, the TYPE I OPD has worse translation fidelity due to the influence of the spurious additional phase $\hat{\varphi}_{0}$; however, the translation to $\hat{\Phi}$ is much linear. An excellent example is given in [29], Fig. 8. 


\section{Reducing the Probability of Jump in TYPE II \\ Estimations}

Despite its multiple benefits, a major drawback of the TYPE II estimation is the demodulation jump, as discussed in Sections 2.B.1 and 2.B.4. Based on the assumption that the PDF of $\overline{\hat{\varphi}_{0}}$ is Gaussian, the theory in Section 2.B.4 predicts the lower limit of the jump probability by Eq. (21), and this limit is reached when the $2 \pi$ phase confinement range is centered on $\varphi_{0}$ and the estimator reaches the phase CRB. It should be noted that the Gaussian PDF of the phase estimation is an essential assumption of the theory, which is demonstrated in Fig. 7. The PDF plotted in the figure was obtained with a real EFPI sensor whose phase was kept constant during the measurement. WLI System I was used in conjunction with the PT method, and the PDF was calculated from 200,000 estimations, which can be well fitted by a Gaussian with $\sigma_{p}=0.158 \pi$.

From the discussion in Sections 2.B.2 and 3.D, the OPD-dependent phase term $\varphi_{0}$ can be calibrated in advance such that during real measurement, the expectation value of $\varphi_{0}$ can be estimated by $\mathrm{OPD}$, resulting in a proper assignment of the $2 \pi$ phase range for $\hat{\varphi}_{0}$ with minimum jump risk.

In detail, after calibration, the $\varphi_{0}$ term is fitted with the OPD using a polynomial as $\varphi_{0}=\mathbf{P}(\mathrm{OPD})$. In real measurement, OPD and $\hat{\varphi}_{0}$ are estimated by a TYPE I estimator (e.g., FFT), and the expectation value of $\varphi_{0}$ is obtained by $\tilde{\varphi}_{0}=\mathbf{P}(\mathrm{OPD})$. Then, multiple $2 \pi$ will be added to or subtracted from $\hat{\varphi}_{0}$ such that it falls within the range $\left[\tilde{\varphi}_{0}-\pi, \tilde{\varphi}_{0}+\pi\right]$. Finally, the TYPE II OPD is estimated by Eqs. (11) and (12).

In Fig. 8 an example is given to illustrate the above process. In Fig. 8(a), the color map of the PDF of the estimated phase is plotted as a function of OPD. The data were acquired experimentally from a sapphirewafer-based EFPI temperature sensor [7]. During the phase calibration, a sufficient amount of data was recorded to capture the OPD $-\varphi_{0}$ relationship (comparing with Fig. 6 reveals reasonable agreement).

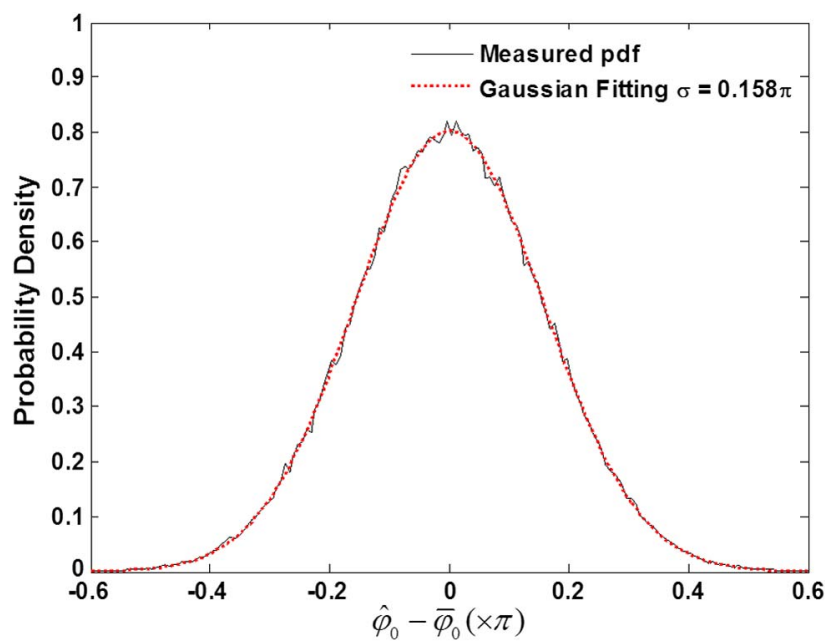

Fig. 7. (Color online) Experimentally obtained PDF of $\varphi_{0}$ estimation.
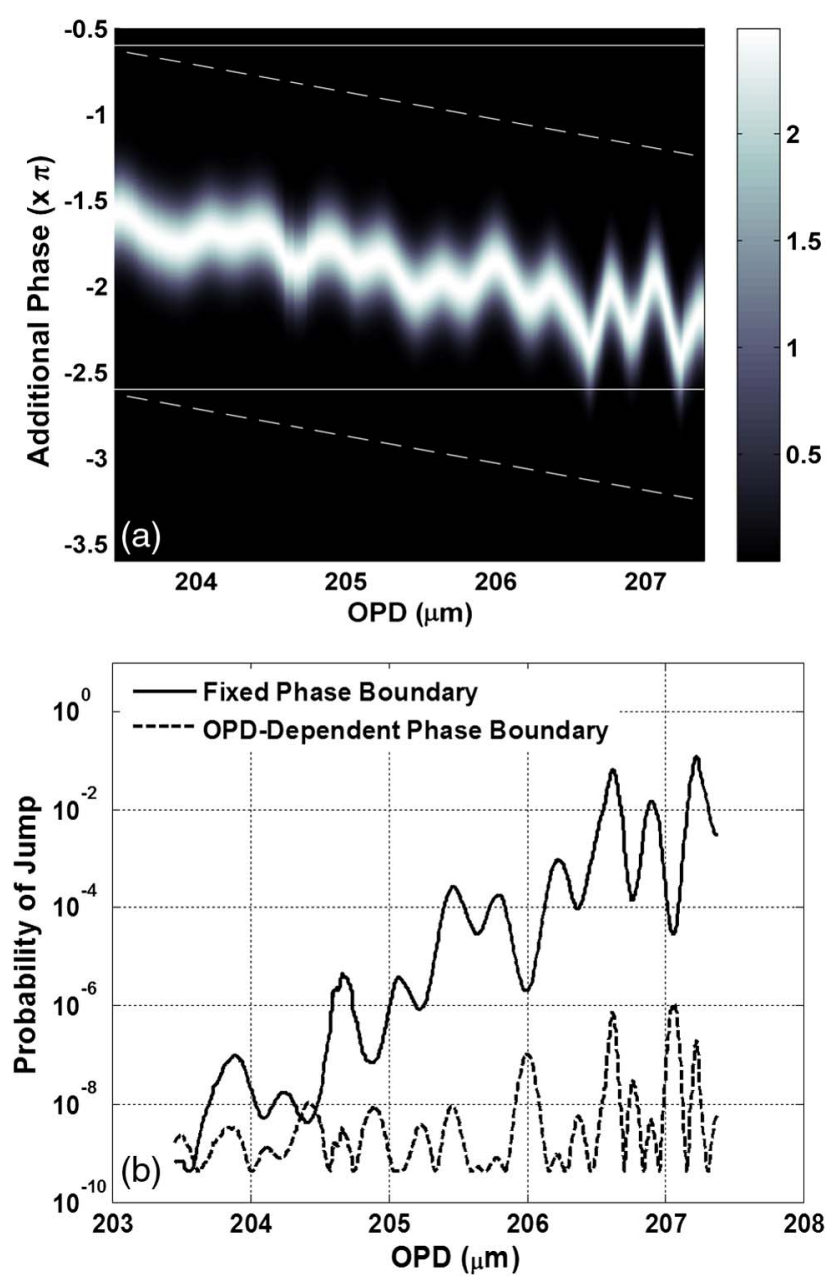

Fig. 8. (Color online) Reduction of jump probability by phase calibration. (a) PDF of $\varphi_{0}$ estimation plotted with OPD, $\sigma_{p}=0.158 \pi$. The area between the solid lines is the assigned phase range assuming constant phase, and dashed lines are the boundaries of the OPD-calibrated range with linear fitting. (b) The corresponding probability of jump. Solid curve: OPD-dependent jump probability for the constant range scheme. Dashed curve: jump probability for the calibrated range scheme.

For simplicity, linear fitting was used to fit the phase with the OPD, and accordingly the phase confinement range was assigned, shown as the dashed lines in Fig. 8(a). In comparison, the solid lines are an alternative range scheme that center at the initial $\varphi_{0}$ but assume constant values. According to the PDF of $\hat{\varphi}_{0}$ and the values of the range boundaries, the probability of jump (i.e., the probability of having phase estimation outside the predefined range) is plotted in Fig. 8(b) for both range schemes. For the fixed phase boundary (solid lines in Fig. 8a), the maximum probability of jump reached $\sim 10 \%$ at $\mathrm{OPD} \approx 207.2 \mu \mathrm{m}$, whereas using the OPD-dependent phase boundary, the jump probability was uniformly below $1 \mathrm{ppm}$. A reduction of jump probability by $10^{5}$ was resulted.

\section{Conclusion}

The demodulation of the WLI low-finesse FP sensor is identical to parameter estimation of a 
noise-contaminated, real, discrete tone with finite length. This forces us to solve the problem, and to evaluate the estimators in the context of harmonic analysis. We call attention to the importance of the CRBs, because any developed algorithms should be evaluated using the bounds as standards. Three algorithms are compared in terms of their errors (including estimation variance and bias) and complexities; comparisons with the CRBs are highlighted.

In spite of its similarity to traditional harmonic analysis, the WLI problem bears some unique features. The data are real and have finite length and low frequency, and consequently the bias caused by spectral leakage becomes dominant when the SNR is high. A solution is to use windows with effective sidelobe suppression in the periodogram, at the expense of moderately increased variance.

The TYPE I estimation (denoted as OPDD in the paper) will suffice if the requirement for resolution is not high. Despite low resolution, this approach has no jump problem. If high resolution is desired, both OPPD and $\hat{\varphi}_{0}$ need to be estimated and subsequently combined to yield the TYPE II estimation ( $\left(\hat{P} D_{\text {tot }}\right)$. $O \hat{P} D_{\text {tot }}$ has significantly reduced estimation error and demodulation nonlinearity; however, it has the risk of abrupt demodulation jumps. The jump is attributed to the $\hat{\varphi}_{0}$ term, the value of which is intrinsically ambiguous (with $2 \pi$-step jumps). Moreover, the value of $\varphi_{0}$ is not a constant and can vary during measurement. As such, $\hat{\varphi}_{0}$ and OPDD are of parallel importance in TYPE II estimation. If $\varphi_{0}$ is reliably dependent on OPD, it can be calibrated in advance and an appropriate (OPD-dependent) $2 \pi$ range can be assigned to $\hat{\varphi}_{0}$ to minimize the occurrence of jumps.

\section{Appendix A: Noise Reduction Mechanism of TYPE II Estimations}

In Section 2.B.3, we have shown the noise reduction gain in Eq. (17). In this appendix we will explain more on the noise reduction mechanism of the TYPE II approaches.

Taking the variance on both sides of Eq. (18), we get

$$
\operatorname{var}[\hat{\Phi}]=k_{c}^{2} \cdot \operatorname{var}[\hat{L}]+2 k_{c} \cdot \operatorname{cov}[\hat{L}, \hat{\varphi}]+\operatorname{var}[\hat{\varphi}],
$$

where $\operatorname{cov}[\bullet]$ denotes the covariance $\hat{L}$ and $\hat{\varphi}$ are estimations of the OPD and additional phase. The values of the terms on the right-hand side of Eq. (A1) can be obtained from the components of the inversed Fisher information matrix, and correspondingly labeled as $J^{(i, j)}(i, j=1,2)$. The values of $\operatorname{var}[\hat{L}]=J^{(11)}$ and $\operatorname{var}[\hat{\varphi}]=J^{(22)}$ are defined in Eqs. (14) and (16), respectively. The value of $\operatorname{cov}[\hat{L}, \hat{\varphi}]$ is determined by the antidiagonal elements $J^{(1,2)}=J^{(2,1)}$, which can be calculated as

$$
J^{(1,2)}=J^{(2,1)}=-\frac{6\left(2 n_{0}+N-1\right)}{S \cdot \Delta k \cdot N\left(N^{2}-1\right)}
$$

The value is negative and sets the lower limit of the covariance in the sense that $|\operatorname{cov}[\bullet]| \geq\left|J^{(1,2)}\right|$. To estimate the variance of $\hat{\Phi}$, we insert Eqs. (14), (16), and (2) into Eq. (1) and get

$$
\operatorname{var}[\hat{\Phi}] \approx \frac{12 k_{c}^{2} N-12 k_{c} \Delta k\left(2 n_{0}+N-1\right) N+12 \Delta k^{2} Y}{S \cdot \Delta k^{2} \cdot N^{2}\left(N^{2}-1\right)} .
$$

In order to simplify Eq. (A3), the following approximations are made: $P \approx \frac{N^{2}}{2}, Q \approx \frac{N^{3}}{3}, k_{c} \approx k_{0}+\frac{1}{2} N \Delta k=$ $\left(n_{0}+\frac{1}{2} N\right) \Delta k, k_{c}^{2} \approx \frac{Y \Delta k^{2}}{N}-\frac{(N \Delta k)^{2}}{12}$, which lead to the following approximation:

$$
Y \Delta k^{2} \approx\left[1+\frac{1}{12}\left(\frac{N}{n_{0}}\right)^{2}\right] k_{c}^{2} N=\left(1+C_{0}\right) k_{c}^{2} N
$$

With the approximations above, Eq. $\underline{\mathrm{A} 3}$ is reduced to

$$
\operatorname{var}[\hat{\Phi}] \approx \frac{1}{S \cdot N}
$$

which is the CRB of $\hat{\varphi}$ with known OPD [Eq. (17)]. We can further prove that the TYPE II OPD estimation [Eq. (12)] approaches the CRB of OPD estimation with known phase by inserting Eqs. (A4) and (12) into Eq. (5) and setting $C_{0}=\left(N / n_{0}\right)^{2} / 120 \approx 0 \overline{\text { (to }}$ demonstrate, putt in parameters for WLI System I, we get $C_{0} \approx 0.01$, and for WLI System II, $C_{0} \approx 0.0001$ ), and finally we get

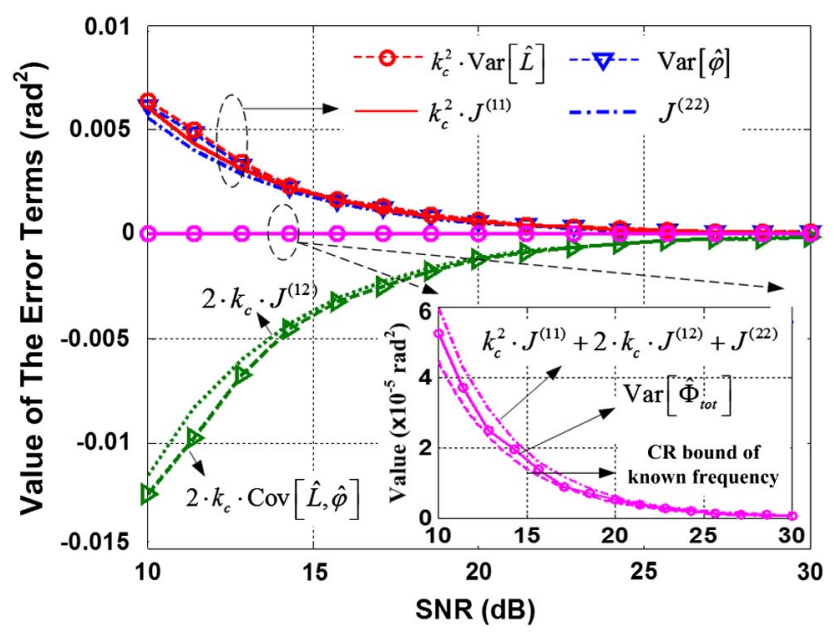

Fig. 9. (Color online) Computer-simulated variance and covariance terms in Eq. (A1). The corresponding CRBs are plotted together. An important observation is that the variance terms and the covariance term cancel to yield a significantly reduced variance for the total phase estimation. 


$$
\operatorname{var}\left[\mathrm{OP} \mathrm{D}_{\mathrm{tot}}\right] \approx \frac{1}{S \cdot(\Delta k)^{2} Y},
$$

which is the CRB for OPD estimation with known phase [Eq. (15)]. The above calculation was confirmed by computer simulation in which the variance and covariance terms in Eq. (A1) are numerically solved $(\mathrm{OPD}=200 \mu \mathrm{m}$, WLI System I with an FFT estimator was used, and at each SNR 1000 observations were made). The result is plotted in Fig. 9. The figure clearly shows how the covariance term cancels the variance terms to yield a much smaller variance for the total phase estimation. It should be noted that Eq. (A5) is the real CRB for the total phase estimation, whereas Eq. (A1) is only an approximation (as shown in the figure, the variance falls in between the two lines).

\section{References}

1. V. Bhatia, K. A. Murphy, R. O. Claus, T. A. Tran, and J. A. Greene, "Recent developments in optical-fiber-based extrinsic Fabry-Perot interferometric strain sensing technology," Smart Mater. Struct. 4, 246-251 (1995).

2. J. Schwider and L. Zhou, "Dispersive interferometric profilometer," Opt. Lett. 19, 995-997 (1994)

3. T. Liu, M. Wu, Y. Rao, D. A. Jackson, and G. F. Fernando, "A multiplexed optical fibre-based extrinsic Fabry-Perot sensor system for in-situ strain monitoring in composites," Smart Mater. Struct. 7, 550-556 (1998).

4. B. Yu, D. W. Kim, J. Deng, H. Xiao, and A. Wang, "Fiber FabryPerot sensors for detection of partial discharges in power transformers," Appl. Opt. 42, 3241-3250, 2003.

5. T. Wei, Y. Han, H.-L. Tsai, and H. Xiao, "Miniaturized fiber inline Fabry-Perot interferometer fabricated with a femtosecond laser," Opt. Lett. 33, 536-538 (2008).

6. M. Born and E. Wolf, Principles of Optics: Electromagnetic Theory of Propagation, Interference and Diffraction of Light, 7th ed. (Cambridge University, 1999).

7. Y. Zhu, Z. Huang, F. Shen, and A. Wang, "Sapphire-fiber-based white-light interferometric sensor for high-temperature measurements," Opt. Lett. 30, 711-713 (2005).

8. K. A. Murphy, M. F. Gunther, A. M. Vengsarkar, and R. O. Claus, "Quadrature phase-shifted, extrinsic Fabry-Perot optical fiber sensors," Opt. Lett. 16, 273-275 (1991).

9. C. Belleville and G. Duplain, "White-light interferometric multimode fiber-optic strain sensor," Opt. Lett. 18, 78-80 (1993).

10. T. Liu and G. F. Fernando, "A frequency division multiplexed low-finesse fiber optic Fabry-Perot sensor system for strain and displacement measurements," Rev. Sci. Instrum. 71, 1275-1278 (2000).

11. S. Taplin, A. G. Podoleanu, D. J. Webb, and D. A. Jackson, "Displacement sensor using channelled spectrum dispersed on a linear CCD array," Electron. Lett. 29, 896-897 (1993).

12. C. Boulet, M. Hathaway, and D. A. Jackson, "Fiber-optic-based absolute displacement sensors at $1500 \mathrm{~nm}$ by means of a variant of channeled spectrum signal recovery," Opt. Lett. 29, 1602-1604 (2004).

13. S. M. Musa, "Real-time signal processing and hardware development for a wavelength modulated optical fiber sensor system," Ph.D. dissertation (Virginia Tech, 1997).

14. A. Majumdar and H. Huang, "Development of an in-fiber white-light interferometric distance sensor for absolute measurement of arbitrary small distances," Appl. Opt. 47, 2821-2828 (2008).

15. T. Liu, D. Brooks, A. R. Martin, R. A. Badcock, and G. F. Fernando, "Design, fabrication, and evaluation of an optical fiber sensor for tensile and compressive strain measurements via the use of white light interferometry," Proc. SPIE 2718, 408-416 (1996).
16. Y. Jiang, "High-resolution interrogation technique for fiber optic extrinsic Fabry-Perot interferometric sensors by the peak-to-peak method," Appl. Opt. 47, 925-932 (2008).

17. M. Takeda, H. Ina, and S. Kobayashi, "Fourier-transform method of fringe-pattern analysis for computer-based topography and interferometry," J. Opt. Soc. Am. 72, 156-160 (1982).

18. J. Yi, "Fourier transform white-light interferometry for the measurement of fiber-optic extrinsic Fabry-Perot interferometric sensors," IEEE Photon. Technol. Lett. 20, 539-541 (2008).

19. V. Bhatia, M. B. Sen, K. A. Murphy, and R. O. Claus, "Wavelength-tracked white light interferometry for highly sensitive strain and temperature measurements," Electron. Lett. 32, 247-249 (1996).

20. B. Qi, G. R. Pickrell, J. Xu, P. Zhang, Y. Duan, W. Peng, Z. Huang, W. Huo, H. Xiao, R. G. May, and A. Wang, "Novel data processing techniques for dispersive white light interferometer," Opt. Eng. 42, 3165-3171 (2003).

21. R. Cortés, A. V. Khomenko, A. N. Starodumov, N. Arzate, and L. A. Zenteno, "Interferometric fiber-optic temperature sensor with spiral polarization couplers," Opt. Commun. 154, 268-272 (1998).

22. F. Shen and A. Wang, "Frequency-estimation-based signalprocessing algorithm for white-light optical fiber Fabry-Perot interferometers," Appl. Opt. 44, 5206-5214 (2005).

23. H. Huang, "Data interrogation for Fabry-Perot white light interferometry," Proc. SPIE 6174, 617419 (2006).

24. X. Zhou and Q. Yu, "Wide-range displacement sensor based on fiber-optic Fabry-Perot interferometer for subnanometer measurement," IEEE Sens. J. 11, 1602-1606 (2011).

25. M. Han, Y. Zhang, F. Shen, G. R. Pickrell, and A. Wang, "Signal-processing algorithm for white-light optical fiber extrinsic Fabry-Perot interferometric sensors," Opt. Lett. 29, 1736-1738 (2004).

26. S. M. Kay, Modern Spectral Estimation: Theory and Application (Prentice-Hall, 1999).

27. Y. J. Rao, X. J. Wang, T. Zhu, and C. X. Zhou, "Demodulation algorithm for spatial-frequency-division-multiplexed fiberoptic Fizeau strain sensor networks," Opt. Lett. 31, 700-702 (2006).

28. H. C. So, Y. T. Chan, Q. Ma, and P. C. Ching, "Comparison of various periodograms for sinusoid detection and frequency estimation," IEEE Trans. Aerosp. Electron. Syst. 35, 945-952 (1999).

29. C. Ma, E. Lally, and A. Wang, "Toward eliminating signal demodulation jumps in optical fiber intrinsic Fabry-Perot interferometric sensors," J. Lightwave Technol. 29, 19131919 (2011).

30. D. Rife and R. Boorstyn, "Single tone parameter estimation from discrete-time observations," IEEE Trans. Inf. Theory 20, 591-598 (1974).

31. F. J. Harris, "On the use of windows for harmonic analysis with the discrete Fourier transform," Proc. IEEE 66, 51-83 (1978).

32. H. C. So, C. Kit Wing, Y. T. Chan, and K. C. Ho, "Linear prediction approach for efficient frequency estimation of multiple real sinusoids: algorithms and analyses," IEEE Trans. Signal Process. 53, 2290-2305 (2005).

33. M. Han and A. Wang, "Mode power distribution effect in white-light multimode fiber extrinsic Fabry-Perot interferometric sensor systems," Opt. Lett. 31, 1202-1204 (2006).

34. C. Ma, B. Dong, J. Gong, and A. Wang, "Decoding the spectra of low-finesse extrinsic optical fiber Fabry-Perot interferometers," Opt. Express 19, 23727-23742 (2011).

35. C. Ma and A. Wang, "Multimode excitation-induced phase shifts in intrinsic Fabry-Perot interferometric fiber sensor spectra," Appl. Opt. 49, 4836-4845 (2010).

36. G. Ghosh, M. Endo, and T. Iwasaki, "Temperature-dependent Sellmeier coefficients and chromatic dispersions for some optical fiber glasses," J. Lightwave Technol. 12, 1338-1342 (1994).

37. T. H. Barnes, "Photodiode array Fourier transform spectrometer with improved dynamic range," Appl. Opt. 24, 3702-3706 (1985). 